\title{
Onsager reciprocity relations without microscopic reversibility
}

\author{
D. Gabrielli \\ SISSA, Scuola Internazionale Superiore di Studi Avanzati \\ Via Beirut 2-4, 34014 Trieste, Italia \\ G. Jona-Lasinio \\ Dipartimento di Fisica, Università di Roma "La Sapienza" \\ Piazza A. Moro 2, 00185 Roma, Italia \\ C. Landim \\ IMPA, Estrada Dona Castorina 110 \\ J. Botanico, 22460 Rio de Janeiro RJ, Brasil \\ and LAMS de l'Université de Rouen, Faculté de Sciences \\ BP 118,F-76134 Mont-Saint-Aignan Cedex, France
}

In this paper we show that Onsager-Machlup time reversal properties of thermodynamic fluctuations and Onsager reciprocity relations for transport coefficients can hold also if the microscopic dynamics is not reversible. This result is based on the explicit construction of a class of conservative models which can be analysed rigorously.

Fundamental contributions to the theory of irreversible processes were the derivation of the reciprocal relations for transport coefficients in states deviating only slightly from equilibrium and the calculation of the most probable trajectory creating a fluctuation near equilibrium. The first result was obtained by Onsager in 1931 [1] and the second one by Onsager and Machlup [2] in 1953. The calculation of the most probable trajectory relies on the reciprocal relations which in turn are a consequence of microscopic reversibility. It turns out that the trajectory in question is just the time reversal of the most probable trajectory describing relaxation to equilibrium of a fluctuation. The latter is a solution to the hydrodynamical équations.

These topics have received a certain amount of attention in the physical litterature in the course of the last forty years. No rigorous results have been however established. More recently, this subjet has been taken up in various papers attempting more rigorous approaches : [3], [4], [5], in the context of so called interacting particle systems and [6], [7] in a context of deterministic dynamical systems.

In [4] we discussed the following question: is microscopic reversibility a necessary condition for the validity of the Onsager and Onsager-Machlup results? The answer to this question is far from obvious because in going from the microscopic to the macroscopic scale a lot of information is lost and irreversibilities at a small scale may be erased when taking macroscopic averages.

In [4] we have exhibited a class of microscopic nonreversible stochastic dynamics for which the time reversal rule of Onsager-Machlup is still valid even for fluctuations very far from equilibrium. This class of dynamics concerns dissipative one component systems with a hydrodynamic equation of gradient type. Therefore there is no Onsager reciprocity relation to verify.

In this paper we present a class of nonreversible multi component conservative models giving rise at the macroscopic level to nonlinear purely diffusive equations in the terminology of [3].

The equations are of the following form

$$
\partial_{t} \rho=\sum_{i=1}^{d} \partial_{u_{i}}\left\{D(\rho) \cdot \partial_{u_{i}} \rho\right\}
$$

where $\rho(u, t)=\left(\rho_{1}(u, t), \ldots, \rho_{n}(u, t)\right)$ is a vector standing for the densities of different kinds of particles and $D$ is in general a nonsymmetric $n \times n$ matrix.

Associated to our models there is an entropy functional $S(\rho)$ that is written as the integral of a density $s(\rho)$ : $S(\rho)=\int s(\rho(u)) d u$.

The Onsager coefficients are defined in this context by

$$
L(\rho)=D(\rho) \cdot R(\rho)
$$

where the matrix $R$ is determined by the entropy density $s(\rho(u))$ in the following way

$$
\left(R^{-1}\right)_{i, j}=\frac{\partial^{2}}{\partial \rho_{i}(u) \partial \rho_{j}(u)} s(\rho(u))
$$

which is by definition a symmetric matrix. Onsager's reciprocity relations mean that $L$ is a symmetric matrix, a property which holds for our models.

In the physical literature one usually proves the Onsager-Machlup time reversal property from Onsager reciprocity relations. In our approach, we follow the opposite order : we obtain a large deviation functional from which we prove the Onsager-Machlup time reversal property and compute the entropy. This in turn allows us to prove Onsager reciprocity relations. Our results are not restricted to the neighborhood of the equilibrium.

For simplicity, we shall restrict ourselves to one dimensional two component models but all analysis can be 
carried out for any space dimension or for any number of components. As in [4], we consider periodic boundary conditions. The systems considered in this paper differ from those of [4] due to the conservative character of the dynamics.

We consider an interacting particle system that describes the evolution of two types of particles on a lattice. The stochastic dynamics can be informally described as follows. Fix a nonnegative function $g: \mathbb{N} \rightarrow \mathbb{R}_{+}$such that $g(0)=0<g(i)$ for $i \geq 1$ and a finite range mean zero transition probability $p(\cdot)$ on $\mathbb{Z}\left(\sum_{y} y p(y)=0\right.$ and $p(x)=0$ for $|x|$ large enough). We shall assume the jump rate $g$ to be Lipschitz and to diverge at the boundary : $|g(k+1)-g(k)| \leq l_{0}$ and $\lim _{k \rightarrow \infty} g(k)=\infty$. If there are $k_{i}, i=1,2$, particles of type $i$ at a site $x$ of $\mathbb{Z}$, at rate $p(y) g\left(k_{1}+k_{2}\right)\left\{k_{i} / k_{1}+k_{2}\right\}$ one particle of type $i$ jumps from site $x$ to $x+y$. This happens independently at each site.

To define precisely the process, we introduce some notation. We shall consider particles evolving on the discrete one dimensional torus with $N$ points, denoted by $\mathbb{Z}_{N}$ (the integers modulo $N$ ). Sites of $\mathbb{Z}_{N}$ are denoted by $x, y$ and the configurations by the greek letter $\eta=\left(\eta_{1}, \eta_{2}\right)$ so that $\eta_{i}(x)$ stands for the total number of particles of type $i$ at site $x$ for the configuration $\eta$.

The generator $L_{N}$ of this Markov process acts on functions $f$ as

$$
L_{N} f=\frac{N^{2}}{2} \sum_{j=1}^{2} \sum_{x, y \in \mathbb{Z}_{N}} p(y) T_{j}^{x, x+y} f
$$

where the addition in $\mathbb{Z}_{N}$ means addition modulo $N$; the operators $T_{1}^{x, y}$ are defined by

$$
\left(T_{1}^{x, y} f\right)\left(\eta_{1}, \eta_{2}\right)=r_{x}(\eta) \eta_{j}(x)\left[f\left(\eta_{1}^{x, y}, \eta_{2}\right)-f\left(\eta_{1}, \eta_{2}\right)\right]
$$

with $r_{x}(\eta)=g\left(\eta_{1}(x)+\eta_{2}(x)\right) /\left\{\eta_{1}(x)+\eta_{2}(x)\right\}$ and $\zeta^{x, y}$ is the configuration obtained from $\zeta$ letting one particle jump from $x$ to $y$ :

$$
\zeta^{x, y}(z)=\left\{\begin{array}{cl}
\zeta(z) & \text { if } z \neq x, y \\
\zeta(z)-1 & \text { if } z=x \\
\zeta(z)+1 & \text { if } z=y
\end{array}\right.
$$

The operators $T_{2}^{x, y}$ are defined in a similar way.

This process has two conserved quantities : the total number of $\eta_{1}$-particles and the total number of $\eta_{2^{-}}$ particles. It is therefore expected that for each fixed density $\rho_{i} \geq 0$ there should exist an equlibrium state with global density of $\eta_{i}$-particles equal to $\rho_{i}$.

To describe these equilibrium probability measures, for each $\varphi_{1}, \varphi_{2} \geq 0$, consider the product probability measure $\nu_{\varphi_{1}, \varphi_{2}}^{N}$ on $\mathbb{N}^{\mathbb{Z}_{N}} \times \mathbb{N}^{\mathbb{Z}_{N}}$ defined by

$$
\begin{gathered}
\nu_{\varphi_{1}, \varphi_{2}}^{N}\left\{(\eta, \xi) ; \eta(x)=k_{1}, \xi(x)=k_{2}\right\} \\
=\frac{1}{Z\left(\varphi_{1}, \varphi_{2}\right)} \frac{\varphi_{1}^{k_{1}} \varphi_{2}^{k_{2}}}{g\left(k_{1}+k_{2}\right) !} \frac{\left(k_{1}+k_{2}\right) !}{k_{1} ! k_{2} !}
\end{gathered}
$$

for $k_{1} \geq 0$ and $k_{2} \geq 0$. In this formula $Z\left(\varphi_{1}, \varphi_{2}\right)$ is a normalizing constant and $g(k)$ ! stands for $g(1) \cdots g(k)$. A simple computation shows that these measures are invariant for the Markov process with generator $L_{N}$ and reversible if and only if the transition probability $p(\cdot)$ is an even function.

Define $\rho_{i}: \mathbb{R}_{+} \times \mathbb{R}_{+} \rightarrow \mathbb{R}_{+}$by $\rho_{i}\left(\varphi_{1}, \varphi_{2}\right)=$ $E_{\nu_{\varphi_{1}, \varphi_{2}}^{N}}\left[\eta_{i}(0)\right]$ and set $\rho^{*}=\rho_{1}+\rho_{2}$. One can check that $\rho^{*}$ is a smooth strictly increasing function of $\varphi_{1}+\varphi_{2}$. Denote by $a=a\left(\rho^{*}\right)$ the inverse of $\rho^{*}: a(\cdot)=\left(\rho^{*}\right)^{-1}(\cdot)$. We have that $\varphi_{i}=\left(\rho_{i} / \rho_{1}+\rho_{2}\right) a\left(\rho_{1}+\rho_{2}\right)$. To keep notation simple we shall denote by $b\left(\rho^{*}\right)$ the function $a\left(\rho^{*}\right) / \rho^{*}$. In conclusion, for each fixed density $\left(\rho_{1}, \rho_{2}\right)$ we obtained an invariant state with total density of $\eta_{i}$-partciles equal to $\rho_{i}$. We shall from now on fix a density $\bar{\rho}=\left(\bar{\rho}_{1}, \bar{\rho}_{2}\right)$.

Let us consider now the unit interval $\mathbb{T}=[0,1)$ with periodic boundary condition and functions $\gamma_{i}: \mathbb{T} \rightarrow \mathbb{R}_{+}$, $i=1,2$ with global density $\bar{\rho}_{i}: \int_{\mathbb{T}} \gamma_{i}(u) d u=\bar{\rho}_{i}$. The main object of our study is the empirical density $\mu^{N}(t)=$ $\left(\mu_{1}^{N}(t), \mu_{2}^{N}(t)\right):$

$$
\mu_{i}^{N}(t, x)=\frac{1}{N} \sum_{y \in \mathbb{Z}_{N}} \eta_{i}(t, y) \delta\left(x-\frac{y}{N}\right) .
$$

If we denote by $Q_{\gamma_{1}, \gamma_{2}}^{N}$ the distribution law of the tragectories $\mu^{N}(t)$ when the initial measure is concentrated on a configuration pair $\left(\eta_{1}^{N}, \eta_{2}^{N}\right)$ such that $\mu^{N}(0) \rightarrow$ $\left(\gamma_{1}(u) d u, \gamma_{2}(u) d u\right)$ as $N \uparrow \infty$, it is possible to show that $Q_{\gamma_{1}, \gamma_{2}}^{N}$ converges weakly as $N$ goes to infinity to the measure concentrated on the path $\rho(u, t)$ that is the unique solution of

$$
\left\{\begin{array}{l}
\partial_{t} \rho=\left(\sigma^{2} / 2\right) \partial_{u}\left\{D(\rho) \cdot \partial_{u} \rho\right\} \\
\rho(0, \cdot)=\gamma(\cdot)
\end{array}\right.
$$

In this formula $\sigma^{2}=\sum_{y} y^{2} p(y)$ and $D=D(\rho)$ is the nonsymmetric diffusion matrix given by

$$
D(\rho)=b(\rho) I+b^{\prime}(\rho) J(\rho),
$$

where $I$ is the identity and $J(\rho)$ is the matrix with entries $J_{i, j}(\rho)=\rho_{i}$.

The above result is a law of large numbers that shows that the empirical density in the limit of large $N$ behaves deterministically according to equation (8). We can now ask what is the probability that our system follows a trajectory different from the solution of (8) when $N$ is large but not infinite. This probability is exponentially small in $N$ and can be estimated using the methods of the theory of large deviations introduced for the systems of interest in 9 and 10 . The main idea consists in introducing a modified system for which the trajectory of interest (fluctuation) is typical being a solution of the corresponding hydrodynamic equation, and then comparing the two evolutions. For this purpose, for each 
pair of smooth functions $H_{i}=H_{i}(u, t)$, we consider the Markov process defined by the generator

$$
L_{N, t}^{H} f=\frac{N^{2}}{2} \sum_{j=1}^{2} e^{H_{j}((y+x) / N, t)-H_{j}(x / N, t)} p(y) T_{j}^{x, x+y} f
$$

with $p(\cdot)$ and $T_{j}^{x, x+y}$ as previously defined. The function $H$ can be interpreted as an external field.

The deterministic equation satisfied by the empirical density is now

$$
\left\{\begin{array}{l}
\partial_{t} \rho=\left(\sigma^{2} / 2\right) \partial_{u}\left\{D(\rho) \cdot \partial_{u} \rho\right\}-\sigma^{2} \partial_{u}\{b(\rho) A(\rho, H)\} \\
\rho(0, \cdot)=\gamma(\cdot)
\end{array}\right.
$$

where $A(\rho, H)$ is the vector with components $A_{i}=$ $\rho_{i} \partial_{u} H_{i}$.

Given a function $\rho(u, t)$ twice differentiable with respect to $u$ and once with respect to $t$ and such that $\int_{T} \rho_{i}(u, t) d u=\bar{\rho}_{i}$ this equation determines uniquely the field $H=\left(H_{1}, H_{2}\right)$.

The probability that the original system follows a trajectory different from a solution of (8) can now be expressed in terms of the field $H$. We introduce the large deviation functional

$$
I_{0, t_{0}}(\rho)=\sum_{i=1}^{2}\left(\sigma^{2} / 2\right) \int_{0}^{t_{0}} d t \int_{\mathbb{T}} d u b(\rho) \rho_{i}\left(\partial_{u} H_{i}\right)^{2}
$$

Let $\mathcal{G}$ be a set of trajectories in the interval $\left[0, t_{0}\right]$. The large fluctuation estimate asserts that

$$
Q_{\gamma_{1}, \gamma_{2}}^{N}(\mathcal{G}) \simeq e^{-N I_{0, t_{0}}(\mathcal{G})}
$$

where

$$
I_{0, t_{0}}(\mathcal{G})=\inf _{\rho \in \mathcal{G}} I_{0, t_{0}}(\rho)
$$

The sign $\simeq$ has to be interpreted as asymptotic equality of the logarithms.

From the equations (12), (13), one sees that to find the most probable trajectory that connects the equilibrium $\bar{\rho}$ to a certain state $\gamma(u)$ one has to find the $\rho(u, t)$ that minimizes $I_{-\infty, 0}(\rho)$ in the set $\mathcal{G}_{\gamma}$ of all trajectories satisfying the boundary conditions

$$
\lim _{t \rightarrow-\infty} \rho(u, t)=\bar{\rho}, \quad \rho(u, 0)=\gamma(u) .
$$

It is now possible to prove, following the same approach of [ 4 , that the unique solution of our variational problem is the function $\rho^{*}(u, t)$ defined by

$$
\rho^{*}(u, t)=\rho(u,-t)
$$

where $\rho(u, t)$ is the solution of the hydrodynamic equation which relaxes to equilibrium with initial state $\gamma$. $\rho^{*}(u, t)$ is therefore a solution of the hydrodynamic equation with inverted drift

$$
\partial_{t} \rho=-\left(\sigma^{2} / 2\right) \partial_{u}\left\{D(\rho) \cdot \partial_{u} \rho\right\} .
$$

Equation (15) is the Onsager-Machlup time-reversal relation.

Denote by $S(\gamma)$ the functional defined by

$$
S(\gamma)=\inf _{\rho \in \mathcal{G}_{\gamma}} I_{-\infty, 0}(\rho)
$$

which, by the Boltzmann-Einstein relationship, has to be identified with the entropy of the system. By inserting (15) in (17) we obtain an explicit formula for the entropy :

$$
S(\gamma)=\int_{\mathbb{T}} s(\gamma(u)) d u
$$

where

$$
s(\gamma)=\sum_{j=1}^{2} E\left(\gamma_{j}(u)\right)+F\left(\gamma_{1}(u)+\gamma_{2}(u)\right)
$$

and $E(\rho)=\int^{\rho} \log \rho^{\prime} d \rho^{\prime}, F(\rho)=\int^{\rho} \log b\left(\rho^{\prime}\right) d \rho^{\prime}$. It is possible to check that $S(\rho(\cdot, t))$ decreases in time if $\rho(\cdot, t)$ is a solution of the hydrodynamic equation ( 8 ).

Of course, the entropy could also be calculated from the equilibrium measure (6) and it is easy to see that the two expressions coincide up to an additive constant.

This explicit expression for the entropy $S(\cdot)$ permits to check Onsager's relations in our model. A simple computation shows that the matrix $R$ defined by equation (3) is such that

$$
\left(R^{-1}\right)_{i, j}=\delta_{i, j} \frac{1}{\gamma_{i}(u)}+\frac{b^{\prime}(\gamma(u))}{b(\gamma(u))}
$$

where $\delta_{i, j}$ stands for the delta of Kronecker and $s(\gamma)$ is the entropy density. The product $L=D R$ can now be computed using the explicit formula for $D$ given in (9) and shown to be a symmetric matrix.

In all the above calculations we never used the symmetry properties of the transition probability $p(\cdot)$ so that they are valid both for reversible and irreversible dynamics.

This provides conclusive evidence that macroscopic reversibility, in the sense of validity of the above results, does not require microscopic reversibility.

\section{ACKNOWLEDGMENTS}

G. J-L. acknowledges a useful correspondence with G. Eyink and interesting discussions with G. Gallavotti. C. L., thanks the INFN (sezione di Roma) and the CNRSCNR agreement for hospitality and support. 
[1] L. Onsager, Phys. Rev. 37 (1931) 405; Phys. Rev. 38 (1931) 2265.

[2] L. Onsager, S. Machlup, Phys. Rev. 91 (1953) 1505; Phys. Rev. 91 (1953) 1512.

[3] G. Eyink, J. Stat. Phys. 61 (1990) 533.

[4] D. Gabrielli, G. Jona-Lasinio, C. Landim and M. E. Vares, Microscopic reversibility and thermodynamics fluctuations (1995), preprint, to appear in proceedings of the conference "Boltzmann's legacy", Rome May 1994.

[5] G. Eyink, J. Lebowitz and H. Spohn, Hydrodynamics and fluctuations outside of local equlibrium: driven diffusive systems (1995), preprint.

[6] G. Gallavotti Chaotic principle : some applications to developed turbulence (1995), preprint.

[7] G. Gallavotti Chaotic hypothesis: Onsager reciprocity and fluctuation-dissipation theorem. (1995), preprint.

[8] G. Jona-Lasinio, C. Landim, M. E. Vares, Prob. Theory Rel. Fields 97 (1993) 339.

[9] C. Kipnis, S. Olla, S. R. S. Varadhan, Commun. Pure Appl. Math. 42 (1989) 115.

[10] M. Donsker and S. R. S. Varadhan, Commun. Pure Appl. Math. 42 (1989) 243. 